\title{
ENHANCEMENTS TO AN ATMOSPHERIC ASCENT GUIDANCE ALGORITHM
}

\author{
Greg A. Dukeman* \\ NASA Marshall Space Flight Center \\ Huntsville, AL 35812
}

\begin{abstract}
$\underline{\text { Abstract }}$
Enhancements to an advanced ascent guidance algorithm for rocket-powered launch vehicles are described. A general method has been developed for conveniently and efficiently handling the common case of (asymmetric) launch vehicles with unbalanced thrust and aerodynamic moments. The new part of this development concerns the treatment of endo-atmosperic flight. An alternative method for handling the transversality conditions has been developed that eliminates the need for a priori elimination of the constant multipliers that adjoin the terminal state constraints to the performance index. As a result, new constraints can be formulated and implemented with relative ease. The problem of burn-coast-burn trajectory optimization is treated using a modified multiple shooting technique.
\end{abstract}

\section{Nomenclature}

POST

$$
\text { Acronyms }
$$

Program to Optimize Simulated Trajectories

TAEM Terminal Area Energy Management

$A$

$C_{A}$

$C_{N}$

g

H

$\mathrm{h}$

M

$m$

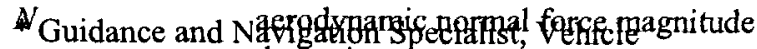
Symbols

aerodynamic axial force magnitude coefficient of axial force coefficient of normal force gravity acceleration vector Hamiltonian function altitude Mach number vehicle mass

Flight Mechanics Exfamic pressure $q \alpha$ product of dynamic pressure and angle of attack

\begin{tabular}{|c|c|}
\hline$q \beta$ & $\begin{array}{l}\text { product of dynamic pressure and } \\
\text { angle of sideslip }\end{array}$ \\
\hline$R$ & great circle range \\
\hline $\mathbf{r}$ & vehicle position vector \\
\hline $\begin{array}{l}\mathrm{r}_{\mathrm{x}}, \mathrm{r}_{\mathrm{y}}, \mathrm{r}_{\mathrm{z}} \\
S\end{array}$ & $\begin{array}{l}\mathrm{x}, \mathrm{y} \text {, and } \mathrm{z} \text { components of position } \\
\text { aerodynamic reference area }\end{array}$ \\
\hline$T$ & thrust magnitude \\
\hline$T_{x}$ & thrust component along $\mathrm{x}$-body axis \\
\hline$T_{z}$ & thrust component along $\mathrm{z}$-body axis \\
\hline$t_{\mathrm{co}}$ & predicted cutoff time from guidance \\
\hline $\mathbf{v}$ & vehicle velocity vector \\
\hline$v_{x}, v_{y}, v_{z}$ & $\mathrm{x}, \mathrm{y}$, and $\mathrm{z}$ components of velocity \\
\hline $\mathbf{x}$ & $\begin{array}{l}\text { column vector containing position } \\
\text { and velocity vectors }\end{array}$ \\
\hline $\mathbf{x}_{\mathbf{b}}, \mathbf{y}_{\mathbf{b}}, \mathbf{z}_{\mathbf{b}}$ & $x-, y-$, and $z$-body axes unit vectors \\
\hline$\alpha$ & vehicle angle of attack \\
\hline$\beta$ & vehicle angle of sideslip \\
\hline$\gamma$ & vehicle flight path angle \\
\hline$\delta$ & $\begin{array}{l}\text { angle between velocity costate vector } \\
\text { and } \mathbf{x}_{\mathbf{b}} \text { vector }\end{array}$ \\
\hline$\phi$ & $\begin{array}{l}\text { angle between position vector and } \\
\text { velocity costate vector }\end{array}$ \\
\hline$\phi_{0}$ & $\begin{array}{l}\text { angle between Earth-relative velocity } \\
\text { vector and velocity costate vector }\end{array}$ \\
\hline$\Delta T$ & actual thrust minus vacuum thrust \\
\hline$\lambda$ & $\begin{array}{l}\text { column vector containing position } \\
\text { and velocity costates }\end{array}$ \\
\hline$\lambda_{\mathbf{r}}$ & position costate \\
\hline$\lambda_{r y}, \lambda_{r z}$ & components of position vector costate \\
\hline$\lambda$ & velocity costate \\
\hline$\sigma_{\mathrm{dir}}$ & $\begin{array}{l}\text { variable set to }+1 \text { or }-1 \text {, specifying } \\
\text { heads-up or heads-down flight, resp. }\end{array}$ \\
\hline$\omega_{0}$ & $\begin{array}{l}\text { parameter associated with invocation } \\
\text { of linear gravity field assumption }\end{array}$ \\
\hline & Subscripts \\
\hline $1, I$ & $\begin{array}{l}\text { vector or quantity relative to the } \\
\text { inertial frame }\end{array}$ \\
\hline $\boldsymbol{r}, \boldsymbol{r}$ & $\begin{array}{l}\text { vector or quantity relative to the } \\
\text { Earth-fixed frame }\end{array}$ \\
\hline 0 & initial \\
\hline
\end{tabular}




\section{Introduction}

Guidance algorithms for future reusable launch vehicles must be very adaptive and robust and they must fly near-optimal trajectories in order to meet challenging cost, reliability, and safety goals. For launch to a circular low-Earth orbit, the mission is performed using either a direct insertion (single burn) into orbit or by using a burn-coast-burn sequence.. Theoretically, executing two burns, i.e., the burn-coastburn sequence, is more efficient, with respect to fuel usage, than is the single burn direct insertion. One way of executing the burn-coast-burn sequence is to a priori compute the fully-optimized trajectory and extract the optimal first-burn cutoff targets and the coast duration from the optimal trajectory. These can then be loaded onto the onboard computer so that the onboard guidance need be concerned with optimizing only a portion (one burn) of the overall trajectory at any given time. This works well and is optimal under the assumption of perfect modeling of the vehicle and environment. An increase in vehicle autonomy and performance can be attained by incorporating burncoast-burn optimization capability into the onboard guidance.

Brown, et. al. ${ }^{1}$, and Cohen and Brown ${ }^{2}$ did some of the earliest research on multi-burn trajectory optimization applied to real-time guidance, using optimal control theory. Jezewski ${ }^{3}$ extended the previous work using a linear gravity field approximation to reduce the computation time required to optimize multi-burn trajectories. Hardtla, ${ }^{4}$ developed the (production) multi-burn optimization 'Gamma guidance' system for the IUS spacecraft. Unlike the algorithms described above, Gamma guidance used the impulsive burn assumption. Gath and Calise ${ }^{5}$ developed multi-burn optimization capability into a hybrid ascent guidance algorithm.

In the atmospheric guidance research of Refs. ${ }^{67} 89$ it was assumed in the formulations that the thrust was always directed along the $\mathrm{x}$-body axis which is approximately true for symmetric vehicles like the Saturn V but not for asymmetric vehicles like the space shuttle. The more general case of time-varying thrust direction in the $x-z$ body axes plane, applicable to asymmetric vehicles, is treated here.

In all the guidance research using principles of optimal control theory 1,2,3,5,6,7,8,9, transversality conditions were handled by analytically eliminating the constant multipliers that adjoin the terminal state constraints to the cost. Each distinct set of terminal state constraints (each corresponding to a particular mission objective, e.g., circularization, cutoff at specified altitude and speed, cutoff at specified inclination and semi-major axis, etc.) requires independent analyses (in general) to eliminate the multipliers. A motivation for (analytically) eliminating the multipliers a priori is that it probably results in slightly less computation than numerical elimination. The motive for numerical elimination is that new terminal state constraint sets can be formulated with relative ease. A numerical approach is presented here that works well and incurs very little additional computational burden over the analytical method.

The rest of this paper is organized as follows. The next section describes the trajectory optimization problem associated with ascent guidance. Next, the additional necessary conditions associated with the multi-burn optimization formulation are presented. The modifications for handling asymmetric vehicles are discussed next followed by a discussion of the numerical method for handling transversality conditions. Numerical results are then given using the guidance formulations. The paper is ended with conclusions and recommendations.

\section{Trajectory Optimization Problem}

The equations of motion for a thrusting rocket in atmospheric flight are:

$$
\begin{aligned}
& \dot{\mathbf{r}}=\mathbf{v} \\
& \dot{\mathbf{v}}=\mathbf{g}+\frac{\left(T_{x}-A\right) \mathbf{x}_{\mathbf{b}}+\left(T_{z}-N\right) \mathbf{z}_{\mathbf{b}}}{m}
\end{aligned}
$$

where the thrust components, $T x$ and $T z$, and the axial and normal forces, $A$ and $N$ are given by:

$$
\begin{aligned}
& T_{x}=T \cos \delta_{p} \\
& T_{z}=T \sin \delta_{p} \\
& T=T_{v a c}+\Delta T(h) \\
& A=q S C_{A} \quad N=q S C_{N}
\end{aligned}
$$

Some notes on guidance modeling follow. We've assumed that all the thrust is aligned along the $\mathrm{x}$-body axis, $\mathbf{x}_{\mathbf{b}}$. The velocity vector, $\mathbf{v}$, can be taken as the Earth-relative velocity vector or inertial velocity vector depending on the context. For high-speed flight (typically occurring 'outside' the atmosphere), inertial velocity is used whereas for low-speed flight and suborbital missions, it is sufficient to let the velocity in question be Earth-relative velocity. Note that we've assumed the force along the y-body axis is small and hence we ignore it in the equations of motion and consequently in the optimization (but not in the guided simulations). In the proceeding developments, the position and velocity vectors will be expressed in the guidance coordinate frame illustrated in Figure 1. The guidance coordinate frame is an Earth-centered, right- 
handed, inertial coordinate system with the $x$-axis aligned with the local vertical and the $z$-axis aligned along the expected downrange direction. It is redefined each guidance cycle with the vehicle's current latitude, longitude and an azimuth angle which approximates the downrange direction of travel. This is a convenient frame to work in because, for example, the initial position vector expressed in the guidance frame has $y$ - and z-components equal to zero and the $y$ components of position and velocity are typically near zero.

The aerodynamic coefficients are modeled using least squares polynomial coefficients interpolated with cubic spline functions of Mach number, a common technique for reduction of aerodynamics coefficient data in trajectory optimization. The thrust difference term, $\Delta T$, due to the effects of the atmosphere on thrust, can in general be represented by a cubic spline function. The density, $\rho$, is represented by a least squares curve fit of the standard atmosphere and matches the latter to within 5 percent up to $70 \mathrm{~km}$. Similarly, a least squares $3^{\text {rd }}$-order polynomial fit is used to accurately model the speed of sound, $a$.

At the time of engine cutoff, $t_{c o}, k$ terminal state constraints, nonlinear functions of the states, are imposed:

$$
\begin{aligned}
& \psi_{i}=\psi_{i}\left(\mathbf{r}\left(t_{\text {Cutoff }}\right), \mathbf{v}\left(t_{\text {Cutoff }}\right)\right)=0, \\
& i=1, \ldots, k, \quad k \leq 6
\end{aligned}
$$

Examples of terminal constraints include final position magnitude, flight path angle, semi-major axis, argument of perigee, inclination and longitude of the ascending node.

In general, path constraints of the form:

$$
S(\mathbf{x}, \mathbf{u}, t) \leq 0
$$

are imposed where $\mathbf{x}$ is the state and $\mathbf{u}$ is the control. Examples include maximum axial and normal acceleration, maximum normal force, minimum throttle level, angle of attack, angle of sideslip, and $\min / \max$ values of $q \alpha, q \beta$ and maximum dynamic pressure. The axial force and minimum throttle constraints are fairly straightforward to handle. Gath and Calise considered angle of attack and normal force constraints in Ref. Error! Bookmark not defined. using the mathematical rigor contained in Ref. 10, section 3.10. Constraints on $q \alpha$ and $q \beta$ can be handled in an analogous way because they too are functions of the state and control variables. The maximum dynamic pressure constraint, however, is a state variable constraint. It is well-known that state variable inequality constraints are difficult to treat using optimal control theory. One can also easily conceive of alternative, less rigorous methods of constraining dynamic pressure that may be just as effective and more appropriate for onboard guidance, e.g., Corvin ${ }^{11}$ uses a feedback control law which provides maximum dynamic pressure control via throttle modulation.

We consider now the question of where to point the $z$-body axis, $\mathbf{z}_{\mathrm{b}}$, which is a function of the control $\mathbf{x}_{\mathbf{b}}$ but is not fully specified given $\mathbf{x}_{\mathbf{b}}$. This decision is a function of how we want the vehicle to fly. We can choose to construct $\mathbf{z}_{\mathbf{b}}$ so that the vehicle flies at zero angle of sideslip:

$$
\mathbf{z}_{\mathbf{b}} \| \mathbf{x}_{\mathbf{b}} \times\left(\mathbf{x}_{\mathbf{b}} \times \mathbf{v}\right)
$$

or so the vehicle flies a zero degree ("heads-up) or 180 degree ("heads-down") bank angle trajectory:

$$
\mathbf{z}_{\mathbf{b}} \| \mathbf{x}_{\mathbf{b}} \times\left(\mathbf{x}_{\mathbf{b}} \times \mathbf{r}_{\mathbf{0}}\right)
$$

The zero-sideslip option requires a non-zero roll angle and possibly excessive roll maneuvering. It is likely that future launch vehicles will have limited roll control authority. The heads-up/heads-down option will inherently result in larger angles of sideslip but this can be attenuated fairly easily, if need be, by imposing a sideslip path constraint. In the proceeding, we adopt the heads-up/heads-down option.

The optimization problem can be stated as follows. Determine the $\mathrm{x}$-body axis history, $\mathbf{x}_{\mathrm{b}}(\mathrm{t})$, that maximizes the final vehicle mass (equivalent to minimizing fuel usage or minimizing flight time) subject to the equations of motion (1), the terminal constraints (3) and the path constraints (4)

\section{Costate Differential Equations}

We note that the atmospheric portion of flight occurs over a very small ground track, enabling the use of the flat-Earth approximations ${ }^{12}$ :

$$
\begin{gathered}
h \cong r_{x}-r_{E} \\
\mathbf{g} \cong\left[\begin{array}{lll}
g_{0} & 0 & 0
\end{array}\right]^{T}
\end{gathered}
$$

With these, the state equations become (with explicit state dependencies called out): 


$$
\begin{aligned}
& \dot{r}_{x}=v_{x} \quad \begin{array}{l}
\dot{r}_{y}=v_{y} \quad \dot{r}_{z}=v_{z} \\
\left(T\left(r_{x}\right)-A\left(r_{x}, \mathbf{v}\right)\right) x_{b x_{x}}-N\left(r_{x}, v\right) z_{b x_{x}} \\
m
\end{array} \\
& \dot{v}_{x}=-g_{0}+\frac{\left(T\left(r_{x}\right)-A\left(r_{x}, \mathbf{v}\right)\right) x_{b y_{x}}-N\left(r_{x}, \mathbf{v}\right) z_{b y}}{m} \\
& \dot{v}_{y}=\frac{\left(T\left(r_{x}\right)-A\left(r_{x}, \mathbf{v}\right)\right) x_{b z}-N\left(r_{x}, \mathbf{v}\right) z_{b z}}{m}
\end{aligned}
$$

The costate equations then are given by:

$$
\begin{aligned}
& \dot{\lambda}_{r_{y}}=\dot{\lambda}_{r_{z}}=0 \\
& \dot{\lambda}_{r x}=-\frac{\partial H}{\partial r_{x}}=\frac{-\left(T_{h}-A_{h}\right) \lambda_{\mathbf{v}} \cdot \mathbf{x}_{\mathbf{b}}+N_{h} \lambda_{\mathbf{v}} \cdot \mathbf{z}_{\mathbf{b}}}{m} \\
& \dot{\lambda}_{v x}=-\frac{\partial H}{\partial v_{x}}=-\lambda_{r x}+\frac{A_{v x} \lambda_{\mathbf{v}} \cdot \mathbf{x}_{\mathbf{b}}+N_{v x} \lambda_{\mathrm{v}} \cdot \mathbf{z}_{\mathbf{b}}}{m} \\
& \dot{\lambda}_{v y}=-\frac{\partial H}{\partial v_{y}}=-\lambda_{r y}+\frac{A_{v y} \lambda_{\mathbf{v}} \cdot \mathbf{x}_{\mathbf{b}}+N_{v y} \lambda_{\mathbf{v}} \cdot \mathbf{z}_{\mathbf{b}}}{m} \\
& \dot{\lambda}_{v z}=-\frac{\partial H}{\partial v_{z}}=-\lambda_{r z}+\frac{A_{v z} \lambda_{\mathbf{v}} \cdot \mathbf{x}_{\mathbf{b}}+N_{v z} \lambda_{\mathrm{v}} \cdot \mathbf{z}_{\mathbf{b}}}{m}
\end{aligned}
$$

where the subscripts $h, v x, v y$, and $v z$ denote partial differentiation with respect to those variables. No known analytic solutions for the atmospheric state/costate system exist so we resort to a second-order Runge-Kutta numerical integration scheme to propagate the state/costate system. Ten integration steps are sufficient to obtain a good guidance solution.

In the next subsection, we apply the maximum principle to obtain the optimal control for atmospheric flight.

\section{Optimality Condition}

Applying the maximum principle to the Hamiltonian results in the optimization sub-problem:

$$
\max _{\mathbf{x}_{\mathbf{b}}}\left\{\lambda_{v} \cdot\left(\frac{(T-A) \mathbf{x}_{\mathbf{b}}-N \mathbf{z}_{\mathbf{b}}}{m}\right)\right\}
$$

Note that the optimal control, $\mathbf{x}_{\mathbf{b}}{ }^{\circ}$ (and hence, optimal $\mathbf{z}$-body axis, $\mathbf{z}_{\mathbf{b}}{ }^{\circ}$ ) lies in the plane defined by the (initial) position and velocity-costate vectors. Thus, with reference to Figure 2 (after Gath and Calise ${ }^{\text {Error! }}$ Bookmark not defined.), the optimization sub-problem (10) can be written simply as

$$
\max _{\delta}\{(T-A) \cos (\delta)+N \sin (\delta)\}
$$

Note that we need to be able to evaluate $\alpha$ in terms of $\delta$ so that we can evaluate $A$ and $N$ in the previous equation. From Figure 2, it is clear that $\alpha$ is some constant, $\alpha_{0}$, (that is, constant with respect to $\delta$ ) minus $\delta$.

$$
\alpha=\alpha_{0}-\delta
$$

The formula for $\alpha_{0}$ can be derived by setting $\delta$ to zero and solving for $\alpha$ via:

$$
\begin{aligned}
& v_{x_{b}}=\mathbf{v} \cdot \mathbf{x}_{\mathbf{b}}=v \cos \left(\phi_{0}\right) \\
& \mathbf{z}_{\mathbf{b}}=\sigma_{d i r} \frac{\mathbf{x}_{\mathbf{b}} \times\left(\mathbf{x}_{\mathbf{b}} \times \hat{\mathbf{r}}_{\mathbf{0}}\right)}{\sin (\phi)}=\sigma_{d i r} \frac{\cos (\phi) \mathbf{x}_{\mathbf{b}}-\hat{\mathbf{r}}_{0}}{\sin (\phi)} \\
& v_{\mathbf{z}_{b}}=\mathbf{v} \cdot \mathbf{z}_{\mathbf{b}}=\sigma_{d i r} \frac{\left[v \cos (\phi) \cos \left(\phi_{0}\right)-v_{x}\right]}{\sin (\phi)} \\
& \alpha_{0}=\tan ^{-1}\left(\frac{\sigma_{d i r}\left[v \cos (\phi) \cos \left(\phi_{0}\right)-v_{x}\right]}{v \sin (\phi) \cos \left(\phi_{0}\right)}\right)
\end{aligned}
$$

In the preceding, note that $\phi$ and $\phi_{0}$ are simple functions of the state and costate.

The maximization sub-problem can be solved in many ways. One option is to take the derivative with respect to $\delta$, set to zero and use an iterative procedure (e.g., Newton's method) to get the root which corresponds to the optimum $\delta$. This approach was found to be problematic because there are situations when the Hamiltonian (as a function of $\delta$ ) is very flat and Newton's method is very slow to converge. A more direct method is to do a Golden Section search ${ }^{13}$. The Golden Section algorithm is relatively inefficient but the function to be optimized in this case, the Hamiltonian, is fairly inexpensive to evaluate.

Once the Hamiltonian is maximized, we need to construct the $\mathrm{x}$ - and $\mathrm{z}$-body axes so that we can evaluate the state/costate differential equations. Start by expressing the $\mathrm{x}$-body axis as a linear combination of costate and initial position vector

$$
\mathbf{x}_{\mathbf{b}}=a_{1} \hat{\lambda}_{\mathbf{v}}+b \hat{\mathbf{r}}_{0}
$$

Dot the preceding with velocity costate and with initial position to get

$$
\begin{aligned}
& \cos (\delta)=\hat{\lambda}_{\mathbf{v}} \cdot \mathbf{x}_{\mathbf{b}}=a_{1}+b_{1} \cos (\phi) \\
& \cos (\phi+\delta)=\hat{\mathbf{r}}_{\mathbf{0}} \cdot \mathbf{x}_{\mathbf{b}}=a_{1} \cos (\phi)+b_{1}
\end{aligned}
$$

Solve the preceding two equations for $a_{1}$ and $b_{1}$ in terms of the angles $\phi$ and $\delta$ : 


$$
\begin{aligned}
& a_{1}=\frac{\cos (\delta)-\cos (\phi) \cos (\phi+\delta)}{\sin ^{2}(\phi)} \\
& b_{1}=\frac{\cos (\phi+\delta)-\cos (\phi) \cos (\delta)}{\sin ^{2}(\phi)}
\end{aligned}
$$

The $\mathrm{z}$-body axis is constructed via:

$$
\begin{aligned}
& \mathbf{z}_{\mathbf{b}}=\mathbf{x}_{\mathbf{b}} \times \mathbf{y}_{\mathbf{b}}=\frac{\sigma_{d i r}}{\sin (\phi+\delta)} \mathbf{x}_{\mathbf{b}} \times\left(\mathbf{x}_{\mathbf{b}} \times \hat{\mathbf{r}}_{\mathbf{0}}\right) \\
& =\frac{\sigma_{d i r}}{\sin (\phi+\delta)}\left[\begin{array}{c}
\left(a_{1} \cos (\phi)+b_{1}\right)^{2}-1 \\
a_{1} \lambda_{v_{y}}\left(a_{1} \cos (\phi)+b_{1}\right) \\
a_{1} \lambda_{v_{z}}\left(a_{1} \cos (\phi)+b_{1}\right)
\end{array}\right]
\end{aligned}
$$

\section{Transversality Conditions}

The transversality conditions consist of conditions on final costate, referred to in the sequel as 'costate conditions', and on the final Hamiltonian:

$$
\begin{aligned}
& \lambda^{\mathbf{T}}\left(t_{f}\right)=\mathbf{v}^{\mathbf{T}} \boldsymbol{\psi}_{\mathbf{x}} \\
& H\left(t_{f}\right)=1
\end{aligned}
$$

where $v$ is a column-vector of constant Lagrange multipliers, $\psi$ is the column-vector of terminal state constraints and $\Psi_{\mathbf{x}}$ is the 'constraint gradients matrix', that is, the $k$ by 6 matrix whose $k$ rows are the gradients

(wrt state, $\mathbf{x}$ ) of the terminal state constraints. The costate conditions are equivalent to requiring that the final costate vector be orthogonal to the space spanned by all admissible final state variations $\delta \mathbf{x}$. For a given set of $k$ terminal state constraints, expressions for $6-k$ admissible, or 'transversality vectors', are obtained a priori and the inner products of the final costate with

these vectors are iteratively driven to zero (using a modified Newton's method) simultaneously with the terminal state constraints.

Note that, because the costates can be arbitrarily scaled by a positive factor, the end-point condition on the Hamiltonian is equivalent to requiring that the Hamiltonian be positive.

hence, $\left[\mathbf{v}^{\mathrm{T}}, \mathbf{g}(\mathbf{r})^{\mathrm{T}}\right]^{\mathrm{T}}$ is a transversality vector whenever all the terminal constraints are Keplerian constants (also, whenever true anomaly is free) and thus satisfaction of the costate conditions imply that the 'Keplerian' part of the Hamiltonian (at the final time) is zero. Now, note that the sign of the non-Keplerian part of the Hamiltonian is of definite sign:

$$
\lambda_{\mathbf{v}}^{\mathbf{T}} \frac{T}{m} \hat{\mathbf{u}}=\left\|\lambda_{\mathbf{v}}\right\| \frac{T}{m}>0
$$

Hence, $H\left(t_{f}\right)>0$ and the end-point condition on the Hamiltonian can generally be replaced by the simpler (non-constraining) condition that the final costate magnitude be unity.

Typically, for a given mission, a good initial guess for the mission-elapsed time at which dynamic pressure becomes small is available. Up until that 'simulated' time, the atmospheric equations developed earlier are used in the (numerical) propagation of state/costate. At that point, the current propagated state/costate is handed off to the 'vacuum propagator' (after Ref. Error! Bookmark not defined.). It is advantageous to do this, that is, only use the atmospheric equations when absolutely necessary, because the atmospheric equations are much more complicated than the analogous vacuum equations. Note that it is not critical that the predicted time of 'atmospheric exit', $t_{E x o}$, be especially accurate. If need be, the predicted dynamic pressure at $t_{E x o}$ can be monitored and $t_{E x o}$ increased if necessary.

\section{Numerical Solution Method}

The multiple shooting method ${ }^{14}$ is used to solve the resulting two-point boundary-value problem. The values of the six initial costates, and the engine cutoff time are the free variables that must be iterated upon to null the $k$ terminal state constraints, the $6-k$ costate conditions and the constraint on final costate magnitude. Multiple shooting allows the user to guess state/costate values at more than just the initial point, significantly reducing the well-known sensitivity to the initial costates. In fact, it has been found that insertion of just one additional shooting point (besides the initial shooting point), placed just after peak dynamic pressure, dramatically reduces the sensitivity compared to single shooting.

\section{Abort Guidance Formulation}

The abort guidance formulation is very similar to the nominal guidance formulation. In the case of abort to downrange or abort to launch site, the target values are obtained from an entry profile ${ }^{15}$, i.e., a table of reference altitude, speed, and flight path versus rangeto-HAC 'break points', available from onboard data used (or generated) by the entry guidance function. An additional constraint for aborts to a landing site is that fuel be depleted during the ascent burn so that, e.g., landing gear loads are not exceeded. 
A set of terminal constraints for a downrange abort case is given by:

$$
\begin{aligned}
& r-r_{d}=0 \\
& \mathbf{v} \cdot \mathbf{r}-r_{d} v_{d} \sin \left(\gamma_{d}\right)=0 \\
& \mathbf{v} \cdot\left(\left(\mathbf{r} \times \mathbf{r}_{\mathbf{H A C}}\right) \times \mathbf{r}\right)-v_{d} \cos \left(\gamma_{d}\right) r^{2} \sin (\theta)=0 \\
& \mathbf{v} \cdot\left(\mathbf{r} \times \mathbf{r}_{\mathbf{H A C}}\right)=0
\end{aligned}
$$

where the $\mathrm{HAC}$ radius vector, $\mathbf{r}_{\mathrm{HAC}}$, is a unit vector directed from the center of the Earth to the heading alignment cone, the desired values (' $d$ ' subscripts) are obtained from the re-entry profile, and the range angle, $\theta$, is the angle subtended by the vehicle position vector and the HAC radius vector. The first constraint fixes the altitude, the second and third fix the vertical and horizontal speeds, resp., and the fourth nulls the vehicle heading error with respect to the HAC. Note that the third ensures that the vehicle is actually headed toward the $\mathrm{HAC}$ and not away from it.

For the case of downrange aborts, we want to burn all the propellant so, instead of optimizing fuel usage, we choose to maximize final speed $v$.

and

To sum up the constraints, we have the four final state constraints (26), the transversality conditions (33), and the (non-constraining) constraint $\left|\lambda_{\mathrm{v}}\left(t_{j}\right)\right|=1$. The parameters to be iteratively determined are the six initial costates and the time of flight.

\section{Numerical Results}

Will show plots of gimbal, attitude angles and multi-burn trajectories, altitudes, compare performance with single-burn trajectories showing benefits of multiburn optimization. Will compare with POST generated open-loop trajectories.

\section{Summarv and Conclusions}

This paper describes an ascent guidance algorithm that re-optimizes the entire ascent trajectory each guidance cycle from liftoff to main engine cutoff. Highfidelity guided trajectories compared with POST openloop trajectories demonstrate that it provides nearoptimal performance despite flat-Earth simplifications of the costate equations. The high-fidelity trajectory simulator with the guidance cycling at $1 \mathrm{~Hz}$ runs significantly faster than real-time, indicating the algorithm's efficiency. An abort-to-downrange site formulation is given along with guided abort trajectory results.

Future work will involve using day-of-launch winds in the guidance solution and incorporating Machscheduled angle of attack constraints. Several candidate RLVs are 2-stage vehicles with gimbaled engines, possibly requiring modification of the assumption that all thrust is directed along the $\mathrm{x}$-body axis.

\section{Acknowledgement}

\section{References}

${ }^{1}$ Brown, K.R., Harrold, E.F., and Johnson, G.W., "Rapid Optimization of Multiple-Burn Rocket Trajectories", NASA CR-1430, September 1969.

${ }^{2}$ Cohen, A., O., Brown, K. R., "Real-Time Optimal Guidance for Orbital Maneuvering", AIAA Journal, Vol. 11, No. 9, September 1973, pp. 1266-1272.

${ }^{3}$ Jezewski, D.J., "Optimal Analytic Multiburn Trajectories," AIAA Journal, Vol. 10, No.5, 1972, pp. 680-685.

${ }^{4}$ Hardtla, J.W., "Gamma Guidance for the Inertial Upper Stage /IUS/", AIAA PAPER 78-1292, Guidance and Control Conference, Palo Alto, Calif., August 7-9, 1978.

${ }^{5}$ Gath, P., and Calise, A., "Optimization of Launch Vehicle Ascent Trajectories With Path Constraints and Coast Arcs", AIAA Paper 99-4308, August 1999

${ }^{6}$ Brown, K. R., Harrold, E.F., Johnhson, G. W., "Some New Results on Space Shuttle Atmospheric Ascent Optimization", AIAA Paper No. 70-978, 1970.

${ }^{7} \mathrm{Lu}$, P., "Closed-Loop Endo-Atmospheric Ascent Guidance", AIAA Paper 2002-4558.

${ }^{8}$ Dukeman, G., "Atmospheric Ascent Guidance for RocketPowered Launch Vehicles", AIAA Paper 2002-4559.

${ }^{9}$ Calise, A., and Brandt, N., "Generation of Launch Vehicle Abort Trajectories Using a Hybrid Optimization Method", AIAA Paper 2002-4560.

${ }^{10}$ Bryson, Arthur, E., Jr., and Ho, Yu-Chi, "Applied Optimal Control", Hemisphere Publishing Corporation, 1975.

${ }^{11}$ Corvin, M.A., 'Ascent Guidance for a Winged Boost Vehicle', NASA-CR-172083, August 1988.

${ }^{12}$ Vinh, N.X., "Integrals of the Motion for Optimal Trajectories in Atmospheric Flight", AlAA J., 11, (1973), 700-703.

${ }^{13}$ Press, W.H., Teukolsky, S.A., Vetterling, W.T., Flannery, B.P., "Numerical Recipes in C: The Art of Scientific Computing", $2^{\text {nd }}$ ed., Cambridge University Press, 1992.

${ }^{14}$ Stoer, J., and Bulirsch, R., "Introduction to Numerical Analysis", Springer-Verlag, New York, 1980.

${ }^{15}$ Lu, P., Shen, Z., Dukeman, G., Hanson, J., "Entry Guidance by Trajectory Regulation", AIAA paper 2000-3958, Proceedings of Guidance, Navigation and Control Conference, August 14-17, 2000, Denver, CO. 


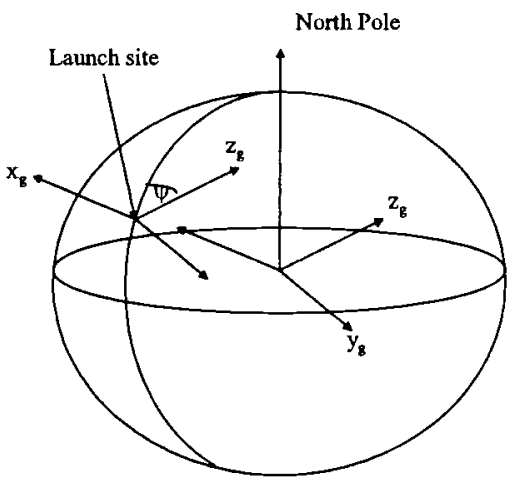

Figure 1: Guidance Reference Frame

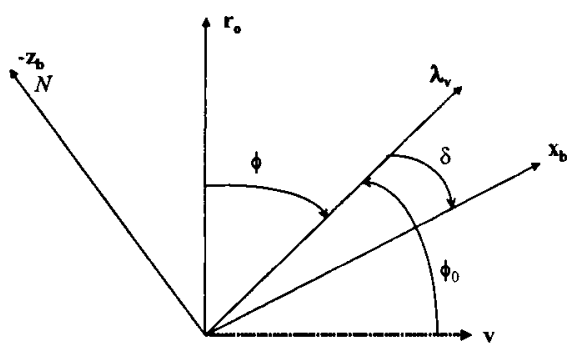

Figure 2: Angles Pertaining To Optimality Condition

Figure 3:

Figure 4:

Figure 14:

Figure 5

Figure 6

Figure 15:

Figure 7

Figure 8

Figure 9:

Figure 10:

Figure 11

Figure 12:

Figure 13: 